\title{
The Total Acoustic Power of a Clamped Circular Plate Located at the Boundary of Three-Wall Corner Region
}

\author{
K. Szemela ${ }^{a, *}$, W.P. RdzaneK ${ }^{a}$ And D. PieczonkA ${ }^{b}$ \\ ${ }^{a}$ Department of Acoustics, Institute of Physics, University of Rzeszów \\ Rejtana 16A, 35-310 Rzeszów, Poland \\ ${ }^{b}$ Stylem, 36-001 Trzebownisko 614, Podkarpackie, Poland
}

\begin{abstract}
The energetic aspect of the sound radiation has been analyzed in the case of the three-wall corner region. This region is the part of space bounded by three baffles arranged perpendicularly to one another. The Neumann boundary value problem has been solved assuming that the sound source is the vibrating circular plate embedded in one of the baffles of the three-wall corner region. The Kelvin-Voigt theory of a visco-elastic plate has been used which allows to include internal attenuation existing in the plate material. It has been assumed that the sound source is excited to vibrations by the external pressure asymmetrically distributed on the plate surface. The modal coefficients of the acoustic impedance have been obtained in the form of the expressions containing single integrals only. The formula describing the acoustic power of the analyzed sound source has been presented as a fourfold infinite series containing the modal coefficients of the acoustic impedance. The influence of some asymmetric excitations on the acoustic power has been analyzed. The possibilities of the modelling some uniform excitations located on the plate fragment of the small area by the point force excitation has been examined. The influence of the transverse baffles on the acoustic power has also been investigated. It has been determined for which frequency the baffles influence on the acoustic power is the greatest.
\end{abstract}

PACS: 43.20.Ks, 43.20.Rz, 43.40.+s, 43.20.-f, 43.20.+g

\section{Introduction}

The plates very often constitute some constructional elements in many industries, transport and architecture. The excited to vibration plates radiate some acoustic wave which can be considered in many cases as undesirable and harmful phenomena. Therefore, the theoretical analysis of the acoustic properties of such source is particularly important from a practical point of view. At the stage of construction, the pure theoretical calculations enable to predict some acoustic properties of designed systems which allows to create and modify projects with view to noise reduction. There are a number of papers devoted to the sound radiation of the plates located in a flat infinite and perfectly rigid baffle. The method of the acoustic resistance matrix has been used to obtain the radiation efficiency of the circular and rectangular plates excited by the harmonic point force excitation for the low frequency range $[1,2]$. Based on the coupling matrix, the acoustic power of rectangular plate has been calculated for the arbitrary boundary conditions [3]. Taking into account the acoustic attenuation and the external surface excitation the formulae describing the sound radiation of the circular membrane [4] and circular plate [5-8] have been obtained. The possibilities of an active reduction of vibrations and noise have been examined in many papers [9-14]. The analysis of the energetic as-

* corresponding author; e-mail: alpha@univ.rzeszow.pl pect of sound radiation is very essential especially when some surface sources generate the acoustic waves into the region bounded by baffles of different shapes and acoustic properties. In particular, the region of the two-wall corner and three-wall corner are very important from a practical point of view. They constitute the part of space bounded by three and two arranged perpendicularly to one another baffles, respectively.

In the low frequency range, the two-wall corner and three-wall corner regions can model commonly found architectural structures for example regions bounded by buildings walls. So far, there are few papers concerning the sound radiation into the two-wall and three-wall corner regions. It concerns even the simplest sound sources such as pistons and membranes. The Green function of the Neumann boundary value problem has been presented for the two-wall corner region as well as for three-wall corner region [15]. In paper [16], the sound pressure radiated by a circular piston has been obtained in the case of the both corner regions. The possibility of the active noise reduction has been examined in the case of two pistons embedded in the different baffles of the three-wall corner [17]. The acoustic power of a vibrating circular membrane located at the boundary of the three-wall corner region has been presented in Ref. [18]. However, there have been obtained the modal coefficients of the acoustic impedance describing interaction of two modes cosine-cosine and sine-sine, only. The interaction of the pair of modes sine-cosine and cosine-sine has not been reported. All the modal coefficients of the acoustic 
impedance needed to obtain the correct results for the acoustic power of the vibrating circular membrane located at the boundary of the two-wall corner region have been presented in Ref. [19]. However, the obtained formulae and performed analysis are insufficient to describe the sound radiation into the three-wall corner region. So far, the radiation of a vibrating circular plate located on one baffle of three-wall corner has not been presented in the case of excitation located on any fragment of the plate surface.

Therefore, this paper is focused on presenting the complete set of the modal coefficients of the acoustic impedance which enable calculation of the acoustic power circular plate located at the boundary of three-wall corner region. The modal coefficients cosine-cosine, sinesine and mixed have been obtained in the form of the integral formulae which are suitable for numerical calculations. The analysis of the acoustic power has been performed for the force point excitation and for excitation located on a fragment of the plate surface. The acoustic power of the circular plate located in a flat baffle has not been analyzed by means of rigorous analysis in the case of excitation located on a plate fragment. However, the solution of this problem, as the limiting case, can be found on the basis of the results presented in this paper.

\section{Analysis assumptions}

The acoustic wave is radiated into the three-wall corner region $\Omega$ bounded by the three perfectly rigid infinite baffles arranged perpendicularly to one another. The sound source is the vibrating circular plate with clamped edge. The plate is located on one of the baffles. The sound radiation is described in the Cartesian coordinates (cf. Fig. 1). The vectors $\boldsymbol{r}=(x, y, z)$ and $\boldsymbol{r}_{s}=\left(x_{s}, y_{s}, 0\right)$ are the leading vectors of the field point and the source point, respectively. The analyzed region of the three-wall corner can be defined as $\Omega=\{0 \leq x<\infty, 0 \leq$ $y<\infty, 0 \leq z<\infty\}$. The location of the plate's centre is determined by $\boldsymbol{l}=\left(l_{x}, l_{y}, 0\right)$ where $|\boldsymbol{l}|>a$ and $l_{x}, l_{y}>a$. Additionally, the local polar coordinates $r_{0}, \varphi_{0}$ have been introduced. The origin of that system is located at the plate centre and its radial axis is parallel to the $y$ axis. The existence of the transverse baffles makes that the plate vibrations should be considered as asymmetric even the excitation is axisymmetric. Moreover, it has been assumed that the transverse deflection amplitude of the plate's points is small enough to analyze them as the linear processes. The plate's surface is excited by the external asymmetric pressure. The influence of some factors derived from outside of the three-wall region has been neglected. The plate's material is assumed to be homogeneous and isotropic. The sound waves are propagated into the homogeneous and lossless light fluid medium. It has been assumed that the analyzed processes are time-harmonic according to the following function: $\exp (-\mathrm{i} \omega t)$, where $\omega$ is the frequency and $\mathrm{i}^{2}=-1$. The acoustic potential satisfies the homogeneous Helmholtz equation into the three-wall corner region

$$
\Delta \Phi(\boldsymbol{r})+k_{0}^{2} \Phi(\boldsymbol{r})=0,
$$

where $\Phi(\boldsymbol{r})$ is the acoustic potential, $k_{0}=2 \pi / \lambda$ denotes the acoustic wavenumber, $\lambda$ is the acoustic wavelength and $\Delta=\partial^{2} / \partial x^{2}+\partial^{2} / \partial y^{2}+\partial^{2} / \partial z^{2}$. Moreover, the Neumann boundary conditions are satisfied at $S_{\Omega}$ :

$$
\begin{aligned}
& \left.\frac{\partial \Phi(\boldsymbol{r})}{\partial x}\right|_{x=0}=\left.\frac{\partial \Phi(\boldsymbol{r})}{\partial y}\right|_{y=0}=0 \\
& \left.\frac{\partial \Phi(\boldsymbol{r})}{\partial z}\right|_{z=0}=\left\{\begin{array}{l}
-v\left(r_{0}, \varphi_{0}\right) \text { on the plate's surface, } \\
0 \text { otherwise. }
\end{array}\right.
\end{aligned}
$$

The solution of Eq. (1) for the whole region $\Omega$ can be obtained after making use of the Sommerfeld radiation conditions

$$
\lim _{r \rightarrow \infty} \Phi(\boldsymbol{r})=0, \quad \lim _{r \rightarrow \infty} r\left[\frac{\partial \Phi(\boldsymbol{r})}{\partial r}+\mathrm{i} k_{0} \Phi(\boldsymbol{r})\right]=0,
$$

which describe the sound radiation at the infinite distance from the source. The considered problem is the classical Neumann boundary value problem. The system of the vibrating source, baffles, acoustic waves radiated into the medium will be further referred to as the vibroacoustic system.

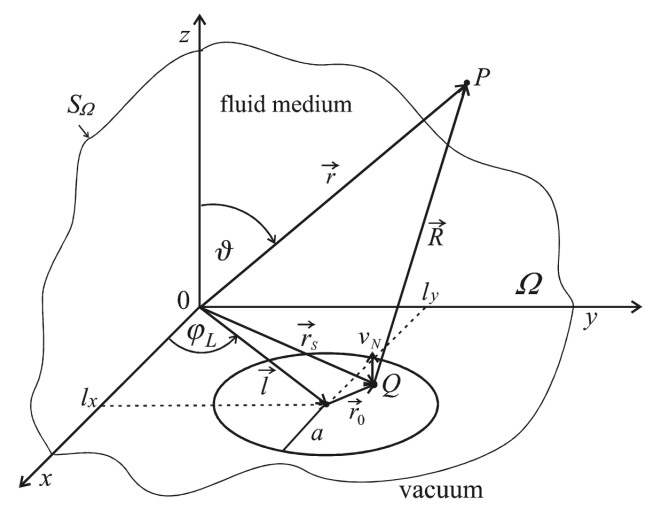

Fig. 1. The three-wall corner region $\Omega$ and the vibrating circular plate located in the baffle $z=0$ where $P=(x, y, z)$ is the acoustic field point and $Q\left(x_{0}, y_{0}, 0\right)$ is the source point.

Based on the Kelvin-Voigt theory of a viscoelastic plate the source vibrations can be described by

$$
\left(k_{\mathrm{T}}^{-4} \nabla^{4}-1\right) W\left(r_{0}, \varphi_{0}\right)+\frac{p\left(r_{0}, \varphi_{0}\right)}{\rho h \omega^{2}}=\frac{f\left(r_{0}, \varphi_{0}\right)}{\rho h \omega^{2}},
$$

where

$$
k_{\mathrm{T}}^{-4}=\frac{D_{\mathrm{T}}}{\rho h \omega^{2}}, \quad D_{\mathrm{T}}=D_{\mathrm{E}}(1-\mathrm{i} \omega \bar{\eta}) .
$$

$W\left(r_{0}, \varphi_{0}\right)$ is the transverse deflection amplitude of the plate points, $p\left(r_{0}, \varphi_{0}\right)$ denotes the acoustic pressure amplitude on the plate surface, $f\left(r_{0}, \varphi_{0}\right)$ is the external excitation, $\rho, h$ are the density of the plate material and the plate thickness, respectively, $D_{\mathrm{E}}=E h^{3} /\left[12\left(1-\nu^{2}\right)\right]$ 
denotes the plate's bending stiffness, $E, \nu$ are the plate Young modulus and the plate Poisson ratio, respectively, $\bar{\eta}$ is the plate's internal attenuation factor $[7,20]$.

The solution of the equation of motion (4) can be presented in the form of double infinite series as follows [20]:

$$
\begin{aligned}
& W\left(r_{0}, \varphi_{0}\right)=\sum_{m=0}^{\infty} \sum_{n=1}^{\infty}\left[c_{m, n}^{(c)} W_{m, n}^{(c)}\left(r_{0}, \varphi_{0}\right)\right. \\
& \left.+c_{m, n}^{(s)} W_{m, n}^{(s)}\left(r_{0}, \varphi_{0}\right)\right],
\end{aligned}
$$

where $W_{m, n}^{(c)}\left(r_{0}, \varphi_{0}\right), W_{m, n}^{(s)}\left(r_{0}, \varphi_{0}\right)$ for $m=0,1,2, \ldots$, $n=1,2,3, \ldots$ constitute the complete eigenfunctions system. The eigenfunctions, in the case of the considered source, take the form of

$$
\begin{aligned}
& \left\{\begin{array}{c}
W_{m, n}^{(c)}\left(r_{0}, \varphi_{0}\right) \\
W_{m, n}^{(s)}\left(r_{0}, \varphi_{0}\right)
\end{array}\right\}=W_{m, n}\left(r_{0}\right)\left\{\begin{array}{c}
\cos m \varphi_{0} \\
\sin m \varphi_{0}
\end{array}\right\}, \\
& W_{m, n}\left(r_{0}\right)=\frac{\sqrt{\varepsilon_{m}}}{\sqrt{2} J_{m}\left(\beta_{m, n}\right)} \\
& \quad \times\left[J_{m}\left(k_{m, n} r_{0}\right)-\frac{J_{m}\left(\beta_{m, n}\right)}{I_{m}\left(\beta_{m, n}\right)} I_{m}\left(k_{m, n} r_{0}\right)\right],
\end{aligned}
$$

where $\varepsilon_{m}=1$ for $m=0, \varepsilon_{m}=2$ for $m>0, k_{m, n}^{2}=$ $\omega_{m, n} \sqrt{\rho h / D_{\mathrm{E}}}, \omega_{m, n}$ is the eigenfrequency of the mode $(m, n), \beta_{m, n}=k_{m, n} a$ denotes the eigenvalue derived from the frequency equation $J_{m+1}\left(\beta_{m, n}\right) I_{m}\left(\beta_{m, n}\right)+$ $J_{m}\left(\beta_{m, n}\right) I_{m+1}\left(\beta_{m, n}\right)=0, a$ is the plate's radius, $c_{m, n}^{(c)}, c_{m, n}^{(s)} \in C$ are constants which have to be found to solve the equation of motion (cf. [8, 21]). The eigenfunctions (7) satisfy the homogeneous equation of motion $[22,23]$

$$
\left(k_{m, n}^{-4} \nabla^{4}-1\right)\left\{\begin{array}{l}
W_{m, n}^{(c)}\left(r_{0}, \varphi_{0}\right) \\
W_{m, n}^{(s)}\left(r_{0}, \varphi_{0}\right)
\end{array}\right\}=0
$$

and have been normalized according to the following relation [20]:

$$
\begin{aligned}
& \int_{0}^{a} \int_{0}^{2 \pi}\left\{\begin{array}{c}
W_{m, n}^{(c)}\left(r_{0}, \varphi_{0}\right) \\
W_{m, n}^{(s)}\left(r_{0}, \varphi_{0}\right)
\end{array}\right\}\left\{\begin{array}{l}
W_{k, l}^{(c)}\left(r_{0}, \varphi_{0}\right) \\
W_{k, l}^{(s)}\left(r_{0}, \varphi_{0}\right)
\end{array}\right\} \\
& \quad \times r_{0} \mathrm{~d} r_{0} \mathrm{~d} \varphi_{0}=S \delta_{m, k} \delta_{n, l},
\end{aligned}
$$

where $S=\pi a^{2}$ is the plate's area, and $\delta_{m, n}$ denotes the Kronecker delta. Applying that the vibration velocity amplitude of the plate points is equal to $v\left(r_{0}, \varphi_{0}\right)=$ $-\mathrm{i} \omega W\left(r_{0}, \varphi_{0}\right)$ and making use of Eq. (6) yields

$$
\begin{aligned}
& v\left(r_{0}, \varphi_{0}\right)=\sum_{m=0}^{\infty} \sum_{n=1}^{\infty} \frac{\omega}{\omega_{m, n}}\left[c_{m, n}^{(c)} v_{m, n}^{(c)}\left(r_{0}, \varphi_{0}\right)\right. \\
& \left.\quad+c_{m, n}^{(s)} v_{m, n}^{(s)}\left(r_{0}, \varphi_{0}\right)\right],
\end{aligned}
$$

where

$$
\left\{\begin{array}{c}
v_{m, n}^{(c)}\left(r_{0}, \varphi_{0}\right) \\
v_{m, n}^{(s)}\left(r_{0}, \varphi_{0}\right)
\end{array}\right\}=v_{m, n}\left(r_{0}\right)\left\{\begin{array}{c}
\cos m \varphi_{0} \\
\sin m \varphi_{0}
\end{array}\right\},
$$

$$
v_{m, n}\left(r_{0}\right)=-\mathrm{i} \omega_{m, n} W_{m, n}\left(r_{0}\right) .
$$

The constants $c_{m, n}^{(c)}$ and $c_{m, n}^{(s)}$ are necessary to obtain the transverse deflection and vibration velocity of the plate points. They depend on the external surface excitation and on the plate's internal attenuation factor. The values of these constants will be found on the basis of equations system which will be later presented in this paper.

\section{Sound pressure}

The sound pressure can be presented by means of the Green function as follows [24]:

$$
\begin{aligned}
p(\boldsymbol{r}) & =-\mathrm{i} k_{0} \rho_{0} c \Phi(\boldsymbol{r}) \\
= & \mathrm{i} k_{0} \rho_{0} c \int_{S} v\left(\boldsymbol{r}_{s}\right) G\left(\boldsymbol{r} \mid \boldsymbol{r}_{s}\right) \mathrm{d} S,
\end{aligned}
$$

where $G\left(\boldsymbol{r} \mid \boldsymbol{r}_{s}\right)$ is the Green function, $c$ is the sound velocity in the medium, $\rho_{0}$ denotes the medium density. Since the baffles are perfectly rigid the integration in above formula can be performed over the source surface, only. The Green function representing the solution of the Neumann boundary value problem for the region of the three-wall corner can be written in the form of (cf. $[15,16])$

$$
\begin{aligned}
& G\left(\boldsymbol{r} \mid \boldsymbol{r}_{s}\right)=\frac{4 \mathrm{i}}{\pi^{2}} \int_{\xi=0}^{\infty} \int_{\eta=0}^{\infty} \cos \xi x \cos \xi x_{s} \cos \eta y \cos \eta y_{s} \\
& \quad \times \exp (\mathrm{i} \gamma z) \frac{d \xi \mathrm{d} \eta}{\gamma}
\end{aligned}
$$

where $\gamma^{2}=k_{0}^{2}-\xi^{2}-\eta^{2}$ and $\xi, \eta, \gamma$ are the Cartesian coordinates of the wavevector. The transformation of the global coordinates into their local counterparts has been introduced (cf. Fig. 1) [17]:

$$
x_{s}=l_{x}+r_{0} \cos \varphi_{0}, \quad y_{s}=l_{y}+r_{0} \sin \varphi_{0} .
$$

Inserting series (10) into Eq. (12) and applying Eqs. (13) and (14) yields the sound pressure in the form of the series of the appropriate modal components.

$$
p(\boldsymbol{r})=\sum_{m=0}^{\infty} \sum_{n=1}^{\infty} \frac{\omega}{\omega_{m, n}}\left[c_{m, n}^{(c)} p_{m, n}^{(c)}(\boldsymbol{r})+c_{m, n}^{(s)} p_{m, n}^{(s)}(\boldsymbol{r})\right],
$$

where

$$
\begin{aligned}
& \left\{\begin{array}{l}
p_{m, n}^{(c)}(\boldsymbol{r}) \\
p_{m, n}^{(s)}(\boldsymbol{r})
\end{array}\right\} \\
& =-\mathrm{i} k_{0} \rho_{0} c \int_{S}\left\{\begin{array}{c}
v_{m, n}^{(c)}\left(r_{0}, \varphi_{0}\right) \\
v_{m, n}^{(s)}\left(r_{0}, \varphi_{0}\right)
\end{array}\right\} G\left(\boldsymbol{r} \mid \boldsymbol{r}_{s}\right) \mathrm{d} S \\
& =\frac{4 k_{0} \rho_{0} c a^{2}}{\pi} \int_{0}^{\infty} \int_{0}^{\infty} \exp (\mathrm{i} \gamma z) \cos \xi x \cos \eta y \\
& \times\left\{\begin{array}{c}
M_{m, n}^{(c)}(\xi, \eta) \\
M_{m, n}^{(s)}(\xi, \eta)
\end{array}\right\} \frac{\mathrm{d} \xi \mathrm{d} \eta}{\gamma},
\end{aligned}
$$




$$
\begin{aligned}
& \left\{\begin{array}{l}
M_{m, n}^{(c)}(\xi, \eta) \\
M_{m, n}^{(s)}(\xi, \eta)
\end{array}\right\}=\frac{-\mathrm{i} \omega_{m, n}}{S} \int_{0}^{a} W_{m, n}\left(r_{0}\right) \\
& \quad \times\left\{\begin{array}{l}
F_{m}^{(c)}\left(\xi, \eta, r_{0}\right) \\
F_{m}^{(s)}\left(\xi, \eta, r_{0}\right)
\end{array}\right\} r_{0} \mathrm{~d} r_{0}, \\
& \left\{\begin{array}{l}
F_{m}^{(c)}\left(\xi, \eta, r_{0}\right) \\
F_{m}^{(s)}\left(\xi, \eta, r_{0}\right)
\end{array}\right\}=\int_{0}^{2 \pi} \cos \left(\xi\left(l_{x}+r_{0} \cos \varphi_{0}\right)\right) \\
& \times \cos \left(\eta\left(l_{y}+r_{0} \sin \varphi_{0}\right)\right)\left\{\begin{array}{c}
\cos m \varphi_{0} \\
\sin m \varphi_{0}
\end{array}\right\} \mathrm{d} \varphi_{0} .
\end{aligned}
$$

After making use of the transformation

$$
\xi=k_{0} \tau \cos \alpha, \quad \eta=k_{0} \tau \sin \alpha, \quad \gamma^{2}=k_{0}^{2}\left(1-\tau^{2}\right),
$$

Eq. (18) can be expressed as

$$
\begin{gathered}
\left\{\begin{array}{c}
F_{m}^{(c)}\left(\tau, \alpha, r_{0}\right) \\
F_{m}^{(s)}\left(\tau, \alpha, r_{0}\right)
\end{array}\right\}=2 \pi \\
\times\left\{\begin{array}{l}
\cos \left(k_{0} \tau l_{y} \sin \alpha\right) \cos \left(k_{0} \tau l_{x} \cos \alpha+m \pi / 2\right) \cos m \alpha \\
-\sin \left(k_{0} \tau l_{y} \sin \alpha\right) \sin \left(k_{0} \tau l_{x} \cos \alpha+m \pi / 2\right) \sin m \alpha
\end{array}\right\} \\
\times J_{m}\left(k_{0} \tau r_{0}\right) .
\end{gathered}
$$

Inserting Eq. (20) into Eq. (17) and applying the frequency equation results in

$$
\begin{gathered}
\left\{\begin{array}{l}
M_{m, n}^{(c)}(\tau, \alpha) \\
M_{m, n}^{(s)}(\tau, \alpha)
\end{array}\right\}=-4 \mathrm{i} \omega_{m, n} \sqrt{\varepsilon_{m}} \\
\times\left\{\begin{array}{l}
\cos \left(k_{0} \tau l_{y} \sin \alpha\right) \cos \left(k_{0} \tau l_{x} \cos \alpha+m \pi / 2\right) \cos m \alpha \\
-\sin \left(k_{0} \tau l_{y} \sin \alpha\right) \sin \left(k_{0} \tau l_{x} \cos \alpha+m \pi / 2\right) \sin m \alpha
\end{array}\right\} \\
\times \psi_{m, n}(\tau),
\end{gathered}
$$

where

$$
\psi_{m, n}(\tau)=\frac{b_{m, n}^{2}}{\sqrt{2} \beta} \frac{\alpha_{m, n} b_{m, n} J_{m}(\beta \tau)-\tau J_{m+1}(\beta \tau)}{b_{m, n}^{4}-\tau^{4}}
$$

and $\alpha_{m, n}=J_{m+1}\left(\beta_{m, n}\right) / J_{m}\left(\beta_{m, n}\right), b_{m, n}=\beta_{m, n} / \beta$, $\beta=k_{0} a$. After changing the coordinates in Eq. (16) according to relations (19), the integral formulations of the sound power modal quantities can be written as

$$
\begin{aligned}
& \left\{\begin{array}{l}
p_{m, n}^{(c)}(\boldsymbol{r}) \\
p_{m, n}^{(s)}(\boldsymbol{r})
\end{array}\right\}=\frac{4 k_{0}^{2} \rho_{0} c a^{2}}{\pi} \int_{0}^{\infty} \int_{0}^{\pi / 2} \exp (\mathrm{i} \gamma z) \\
& \times \cos \xi x \cos \eta y\left\{\begin{array}{l}
M_{m, n}^{(c)}(\tau, \alpha) \\
M_{m, n}^{(s)}(\tau, \alpha)
\end{array}\right\} \frac{\tau \mathrm{d} \tau \mathrm{d} \alpha}{\sqrt{1-\tau^{2}}} .
\end{aligned}
$$

Series (15) together with expressions (23) describes the sound power distribution for the three-wall corner region and can be used for obtaining the acoustic power.

\section{The acoustic power, the acoustic impedance modal coefficients}

The time-averaged acoustic power of the vibrating plate can be obtained on the basis of the formula $[24,25]$

$$
\Pi=\frac{1}{2} \int_{S^{\prime}} p(\boldsymbol{r}) v^{*}(\boldsymbol{r}) \mathrm{d} S^{\prime},
$$

where $S^{\prime}$ is the surface enclosing the sound source, $v^{*}(\boldsymbol{r}) \equiv v^{*}(r, \varphi)$ denotes the conjugate value of $v(r, \varphi)$ given by Eq. (10) and $\boldsymbol{r}=(x, y, 0)$. Applying the impedance approach it has been assumed that the surface $S^{\prime}$ is located directly above the vibrating plate surface. Some medium particles located on the surface $S^{\prime}$ vibrate at the same velocity $v(\boldsymbol{r})$ as the plate. The conjugate value can be written as

$$
\begin{aligned}
& v^{*}\left(r_{0}, \varphi_{0}\right)=\sum_{m=0}^{\infty} \sum_{n=1}^{\infty} \frac{\omega}{\omega_{m, n}}\left[c_{m, n}^{*(c)} v_{m, n}^{*(c)}\left(r_{0}, \varphi_{0}\right)\right. \\
& \left.+c_{m, n}^{*(s)} v_{m, n}^{*(s)}\left(r_{0}, \varphi_{0}\right)\right], \\
& \left\{\begin{array}{l}
v_{m, n}^{*(c)}\left(r_{0}, \varphi_{0}\right) \\
v_{m, n}^{*(s)}\left(r_{0}, \varphi_{0}\right)
\end{array}\right\}=\mathrm{i} \omega_{m, n} W_{m, n}\left(r_{0}\right)\left\{\begin{array}{c}
\cos m \varphi_{0} \\
\sin m \varphi_{0}
\end{array}\right\} .
\end{aligned}
$$

Using Eqs. (25) and (15), the acoustic power can be expressed in the form of the series modal components (cf. [19]):

$$
\begin{aligned}
\Pi & =\sum_{m=0}^{\infty} \sum_{n=1}^{\infty} \sum_{k=0}^{\infty} \sum_{l=1}^{\infty} \frac{\omega^{2}}{\omega_{m, n} \omega_{k, l}}\left[c_{m, n}^{(c)} c_{k, l}^{*(c)} \Pi_{m, n ; k, l}^{(c, c)}\right. \\
& +c_{m, n}^{(c)} c_{k, l}^{*(s)} \Pi_{m, n ; k, l}^{(c, s)}+c_{m, n}^{(s)} c_{k, l}^{*(c)} \Pi_{m, n ; k, l}^{(s, c)} \\
& \left.+c_{m, n}^{(s)} c_{k, l}^{*(s)} \Pi_{m, n ; k, l}^{(s, s)}\right],
\end{aligned}
$$

where the modal coefficient of the acoustic power describing the interaction of cosine modes and sine modes have been defined as

$$
\Pi_{m, n ; k, l}^{(i, j)}=\frac{1}{2} \int_{S^{\prime}} p_{m, n}^{(i)}(\boldsymbol{r}) v_{k, l}^{*(j)}(\boldsymbol{r}) \mathrm{d} S^{\prime}
$$

and $i, j \in\{c, s\}$.

The transformation of the global coordinates of a field point into their local polar counterparts has been introduced

$$
x=l_{x}+r_{0} \cos \varphi_{0}, \quad y=l_{y}+r_{0} \sin \varphi_{0} .
$$

Based on the above relations and Eq. (23), the acoustic power modal components (28) can be formulated in the following form:

$$
\begin{aligned}
& \Pi_{m, n ; k, l}^{(i, j)}=2 k_{0}^{2} \rho_{0} c a^{4} \\
& \quad \times \int_{0}^{\infty} \int_{0}^{\pi / 2} \frac{M_{m, n}^{(i)}(\tau, \alpha) M_{k, l}^{(j)^{*}}(\tau, \alpha) \tau \mathrm{d} \tau \mathrm{d} \alpha}{\sqrt{1-\tau^{2}}},
\end{aligned}
$$

where $i, j \in\{c, s\}$

$$
\begin{aligned}
& \left\{\begin{array}{l}
M_{k, l}^{(c)^{*}}(\tau, \alpha) \\
M_{k, l}^{(s)^{*}}(\tau, \alpha)
\end{array}\right\}=\frac{1}{S} \int_{0}^{a} \int_{0}^{2 \pi}\left\{\begin{array}{l}
v_{k, l}^{(c)^{*}}\left(r_{0}, \varphi_{0}\right) \\
v_{k, l}^{(s)^{*}}\left(r_{0}, \varphi_{0}\right)
\end{array}\right\} \\
& \times \cos \left(k_{0} \tau\left(l_{x}+r_{0} \cos \varphi_{0}\right) \cos \alpha\right)
\end{aligned}
$$




$$
\begin{aligned}
& \times \cos \left(k_{0} \tau\left(l_{y}+r_{0} \sin \varphi_{0}\right) \sin \alpha\right) r_{0} \mathrm{~d} r_{0} \mathrm{~d} \varphi_{0} \\
& =-\left\{\begin{array}{c}
M_{k, l}^{(c)}(\tau, \alpha) \\
M_{k, l}^{(s)}(\tau, \alpha)
\end{array}\right\} .
\end{aligned}
$$

Normalizing the quantities from Eq. (29) by means of the following reference value:

$$
\begin{aligned}
& \Pi_{m, n ; k, l}^{(\operatorname{Re} f .)}=\sqrt{\Pi_{m, n}^{(\operatorname{Re} f .)} \Pi_{k, l}^{(\operatorname{Re} f .)}}=\frac{1}{2} S \rho_{0} c \omega_{m, n} \omega_{k, l}, \\
& \Pi_{m, n}^{(\operatorname{Re} f .)}=\frac{1}{2} \int_{S}\left|v_{m, n}^{(c)}(r, \varphi)\right|^{2} \mathrm{~d} S
\end{aligned}
$$

gives the modal coefficients of the acoustic impedance [24]:

$$
\begin{gathered}
\zeta_{m, n ; k, l}^{(i, j)}=\frac{\Pi_{m, n ; k, l}^{(i, j)}}{\Pi_{m, n, k, l}^{(\operatorname{Re} f .)}}=4 \beta^{2} \sqrt{\varepsilon_{m} \varepsilon_{k}} \\
\quad \times \int_{0}^{\infty} \frac{\phi_{m, k}^{(i, j)}(\tau) \psi_{m, n}(\tau) \psi_{k, l}(\tau) \tau \mathrm{d} \tau}{\sqrt{1-\tau^{2}}},
\end{gathered}
$$

where $i, j \in\{c, s\}$ and

$$
\begin{aligned}
& \phi_{m, k}^{(c, c)}(\tau)=\frac{16}{\pi} \int_{0}^{\pi / 2} \cos ^{2}\left(k_{0} \tau l_{y} \sin \alpha\right) \\
& \quad \times \cos \left(k_{0} \tau l_{x} \cos \alpha+m \pi / 2\right) \\
& \quad \times \cos \left(k_{0} \tau l_{x} \cos \alpha+k \pi / 2\right) \cos m \alpha \cos k \alpha \mathrm{d} \alpha \\
& \phi_{m, k}^{(c, s)}(\tau)=-\frac{8}{\pi} \int_{0}^{\pi / 2} \sin \left(2 k_{0} \tau l_{y} \sin \alpha\right) \\
& \quad \times \cos \left(k_{0} \tau l_{x} \cos \alpha+m \pi / 2\right) \\
& \quad \times \sin \left(k_{0} \tau l_{x} \cos \alpha+k \pi / 2\right) \cos m \alpha \sin k \alpha \mathrm{d} \alpha \\
& \phi_{m, k}^{(s, s)}(\tau)=\frac{16}{\pi} \int_{0}^{\pi / 2} \sin ^{2}\left(k_{0} \tau l_{y} \sin \alpha\right) \\
& \quad \times \sin \left(k_{0} \tau l_{x} \cos \alpha+m \pi / 2\right) \\
& \quad \times \sin \left(k_{0} \tau l_{x} \cos \alpha+k \pi / 2\right) \sin m \alpha \sin k \alpha \mathrm{d} \alpha \\
& \phi_{m, k}^{(s, c)}(\tau)=\phi_{k, m}^{(c, s)}(\tau)
\end{aligned}
$$

Analysis of Eqs. (32) and (33) leads to the following relations:

$$
\begin{aligned}
& \zeta_{m, n ; k, l}^{(c, c)}=\zeta_{m, l ; k, n}^{(c, c)}, \quad \zeta_{m, n ; k, l}^{(s, s)}=\zeta_{m, l ; k, n}^{(s, s)}, \\
& \zeta_{m, n ; k, l}^{(c, c)}=\zeta_{k, n ; m, l}^{(c, c)}, \quad \zeta_{m, n ; k, l}^{(s, s)}=\zeta_{k, n ; m, l}^{(s, s)}, \\
& \zeta_{m, n ; k, l}^{(c, s)}=\zeta_{m, l ; k, n}^{(c, s)}, \quad \zeta_{m, n ; k, l}^{(s, c)}=\zeta_{m, l ; k, n}^{(s, c)}, \\
& \zeta_{m, n ; k, l}^{(c, s)}=\zeta_{k, n ; m, l}^{(s, c)}, \\
& \zeta_{0, n ; k, l}^{(s, s)}=\zeta_{m, n ; 0, l}^{(s, s)}=\zeta_{m, n ; 0, l}^{(c, s)}=\zeta_{0, n ; k, l}^{(s, c)}=0,
\end{aligned}
$$

which can be used to reduce the numerical calculation time. After performing the integration, Eqs. (33) can be written as

$$
\begin{aligned}
& \left\{\begin{array}{l}
\phi_{m, k}^{(c, c)}(\tau) \\
\phi_{m, k}^{(s, s)}(\tau)
\end{array}\right\}=(-1)^{k+m}\left[ \pm J_{k+m}(2 \beta L \tau)\right. \\
& \left.\quad \times \cos \left((k+m) \varphi_{\mathrm{L}}\right)+J_{k+m}\left(2 \beta L_{x} \tau\right)\right] \\
& \quad+(-1)^{k}\left[J_{k-m}(2 \beta L \tau) \cos \left((k-m) \varphi_{\mathrm{L}}\right)\right. \\
& \left.\quad \pm J_{k-m}\left(2 \beta L_{x} \tau\right)\right]+\cos ((k-m) \pi / 2)\left[J_{k+m}\left(2 \beta L_{y} \tau\right)\right. \\
& \left.\quad \pm J_{k-m}\left(2 \beta L_{y} \tau\right)\right]+\delta_{m, k}\left\{\begin{array}{l}
2 / \varepsilon_{m} \\
\operatorname{sgn}(m)
\end{array}\right\}, \\
& \phi_{m, k}^{(c, s)}(\tau)=(-1)^{k+m} J_{k+m}(2 \beta L \tau) \sin \left((k+m) \varphi_{\mathrm{L}}\right) \\
& \quad+(-1)^{k} J_{k-m}(2 \beta L \tau) \sin \left((k-m) \varphi_{\mathrm{L}}\right) \\
& \quad-\sin ((k-m) \pi / 2) \\
& \quad \times\left[J_{k+m}\left(2 \beta L_{y} \tau\right)+J_{k-m}\left(2 \beta L_{y} \tau\right)\right], \\
& \phi_{m, k}^{(s, c)}(\tau)=\phi_{k, m}^{(c, s)}(\tau),
\end{aligned}
$$

where $L=\sqrt{L_{x}^{2}+L_{y}^{2}}=|\boldsymbol{l}| / a>1, L_{x}=l_{x} / a>1$, $L_{y}=l_{y} / a>1, \varphi_{\mathrm{L}}=\arctan \left(L_{y} / L_{x}\right)$ is the angle between the vector $\boldsymbol{l}$ and the $x$-axis (cf. Fig. 1), $\operatorname{sgn}(\cdot)$ denotes the signum function which is equal to $1,0,-1$ where its argument is positive, negative and equal to zero, respectively. All the components in Eq. (35) containing the Bessel function result from the existence of two additional baffles.

Making use of Eqs. (27) and (32), the acoustic power can be presented as the fourfold series of modal coefficients of the acoustic impedance

$$
\begin{aligned}
\Pi & =\frac{1}{2} S \rho_{0} c \omega^{2} \sum_{m=0}^{\infty} \sum_{n=1}^{\infty} \sum_{k=0}^{\infty} \sum_{l=1}^{\infty}\left[c_{m, n}^{(c)} c_{k, l}^{*(c)} \zeta_{m, n ; k, l}^{(c, c)}\right. \\
& +c_{m, n}^{(c)} c_{k, l}^{*(s)} \zeta_{m, n ; k, l}^{(c, s)} \\
& \left.+c_{m, n}^{(s)} c_{k, l}^{*(c)} \zeta_{m, n ; k, l}^{(s, c)}+c_{m, n}^{(s)} c_{k, l}^{*(s)} \zeta_{m, n ; k, l}^{(s, s)}\right] .
\end{aligned}
$$

Applying the above formula requires reducing the infinite series to a finite number of its terms. Consequently, the influence of the higher modes of the plate on its vibrations is neglected. Therefore, the results obtained are the approximation of the real life situation.

\section{Solution to the equation of forced vibrations}

Finding a solution to the equation of motion (4) is equivalent to calculating the constants $c_{m, n}^{(c)}, c_{m, n}^{(s)}$ occurring in the eigenfunctions series (7) for known time-harmonic excitation. Inserting the series (6) into the equation of motion and using Eq. (8) gives 


$$
\begin{aligned}
& \sum_{m=0}^{\infty} \sum_{n=1}^{\infty}\left(\frac{k_{m, n}^{4}}{k_{\mathrm{T}}^{4}}-1\right)\left[c_{m, n}^{(c)} W_{m, n}^{(c)}\left(r_{0}, \varphi_{0}\right)\right. \\
& \left.\quad+c_{m, n}^{(s)} W_{m, n}^{(s)}\left(r_{0}, \varphi_{0}\right)\right]+\frac{p\left(r_{0}, \varphi_{0}\right)}{\rho h \omega^{2}}=\frac{f\left(r_{0}, \varphi_{0}\right)}{\rho h \omega^{2}} .
\end{aligned}
$$

Further applying the standardization condition (9) and the following relation

$$
\int_{0}^{a} \int_{0}^{2 \pi} W_{m, n}^{(c)}\left(r_{0}, \varphi_{0}\right) W_{k, l}^{(s)}\left(r_{0}, \varphi_{0}\right) r_{0} \mathrm{~d} r_{0} \mathrm{~d} \varphi_{0}=0
$$

yields

$$
\begin{aligned}
& \left\{\begin{array}{c}
c_{m, n}^{(c)} \\
c_{m, n}^{(s)}
\end{array}\right\}\left(\frac{k_{m, n}^{4}}{k_{\mathrm{T}}^{4}}-1\right)+\frac{1}{\rho h \omega^{2}}\left\{\begin{array}{c}
P_{m, n}^{(c)} \\
P_{m, n}^{(s)}
\end{array}\right\} \\
& =\frac{1}{\rho h \omega^{2}}\left\{\begin{array}{l}
f_{m, n}^{(c)} \\
f_{m, n}^{(s)}
\end{array}\right\},
\end{aligned}
$$

where

$$
\begin{gathered}
\left\{\begin{array}{c}
P_{m, n}^{(c)} \\
P_{m, n}^{(s)}
\end{array}\right\}=\frac{1}{S} \int_{S} p\left(r_{0}, \varphi_{0}\right)\left\{\begin{array}{l}
W_{m, n}^{(c)}\left(r_{0}, \varphi_{0}\right) \\
W_{m, n}^{(s)}\left(r_{0}, \varphi_{0}\right)
\end{array}\right\} \mathrm{d} S, \\
\left\{\begin{array}{c}
f_{m, n}^{(c)} \\
f_{m, n}^{(s)}
\end{array}\right\}=\frac{1}{S} \int_{S} f\left(r_{0}, \varphi_{0}\right)\left\{\begin{array}{l}
W_{m, n}^{(c)}\left(r_{0}, \varphi_{0}\right) \\
W_{m, n}^{(s)}\left(r_{0}, \varphi_{0}\right)
\end{array}\right\} \mathrm{d} S .
\end{gathered}
$$

Taking into account Eqs. (15), (28) and (32) the equations system $(38)$ can be presented as

$$
\begin{aligned}
& \left\{\begin{array}{l}
c_{m, n}^{(c)} \\
c_{m, n}^{(s)}
\end{array}\right\}\left(\frac{k_{m, n}^{4}}{k_{\mathrm{T}}^{4}}-1\right)-\mathrm{i} \varepsilon_{0} \frac{\omega_{0,1}}{\omega} \\
& \times \sum_{k=0}^{\infty} \sum_{l=1}^{\infty}\left(c_{k, l}^{(c)}\left\{\begin{array}{l}
\zeta_{k, l ; m, n}^{(c, c)} \\
\zeta_{k, l ; m, n}^{(c, s)}
\end{array}\right\}+c_{k, l}^{(s)}\left\{\begin{array}{l}
\zeta_{k, l ; m, n}^{(s, c)} \\
\zeta_{k, l ; m, n}^{(s, s)}
\end{array}\right\}\right) \\
& =\frac{1}{\rho h \omega^{2}}\left\{\begin{array}{c}
f_{m, n}^{(c)} \\
f_{m, n}^{(s)}
\end{array}\right\}
\end{aligned}
$$

where $\varepsilon_{0}=\rho_{0} c / \rho h \omega_{0,1}$ is the dimensionless acoustic attenuation factor. The solution of the above equations system provides the values of constants $c_{m, n}^{(c)}, c_{m, n}^{(s)}$ and enables to obtain the solution to equation of motion of the analyzed sound source. On the basis of Eqs. (34c) and (41), it can be deduced that $c_{0, n}^{(s)}=0$ for $n=1,2,3$. The solutions of Eqs. (41) will be used to calculate the acoustic power on the basis of Eqs. (32) and (36).

\section{Numerical analysis}

The radiation acoustic power of the considered plate has been analyzed for the three different distributions of surface excitation

$$
f_{1}\left(r_{0}, \varphi_{0}\right)=\frac{F_{0}}{r_{0}} \delta\left(r_{0}-\bar{r}\right) \delta\left(\varphi_{0}-\bar{\varphi}\right),
$$

$$
\begin{aligned}
& f_{2}\left(r_{0}, \varphi_{0}\right)=\left\{\begin{array}{ll}
f_{0}, & 0 \leq r_{0} \leq \bar{a} \leq a, \\
0, & 0<\bar{a}<r_{0} \leq a,
\end{array} \quad\right. \text { (42b) } \\
& f_{3}\left(r_{0}, \varphi_{0}\right)= \begin{cases}f_{0}, & \bar{r}-\Delta \bar{r} / 2 \leq r_{0} \leq \bar{r}+\Delta \bar{r} / 2 \\
& \bar{\varphi}-\Delta \bar{\varphi} / 2 \leq \varphi_{0} \leq \bar{\varphi}+\Delta \bar{\varphi} / 2, \\
0, & \text { otherwise, }\end{cases}
\end{aligned}
$$

where $\delta(\cdot)$ is the Dirac delta, $f_{0}[\mathrm{~Pa}]$ is the pressure amplitude, $F_{0}=f_{0} S_{0}[\mathrm{~N}]$ is the force amplitude, $f_{1}\left(r_{0}, \varphi_{0}\right)$ is the plate point excitation located at the point determined by the polar coordinates $(\bar{r}, \bar{\varphi}), f_{2}\left(r_{0}, \varphi_{0}\right)$ denotes the axisymmetric uniform excitation applied to the surface of wheel of radius $\bar{a}=\sqrt{S_{0} / \pi}, f_{3}\left(r_{0}, \varphi_{0}\right)$ is the asymmetric uniform excitation applied to the plate's segment of area $\bar{r} \Delta \bar{r} \Delta \bar{\varphi}$ with its characteristic point $(\bar{r}, \bar{\varphi})$ (cf. Fig. 2), $\Delta \bar{r}=\sqrt{S_{0}}, \Delta \bar{\varphi}=\sqrt{S_{0}} / \bar{r}, S_{0} \ll S$ is the area of surface to which the excitation is applied.

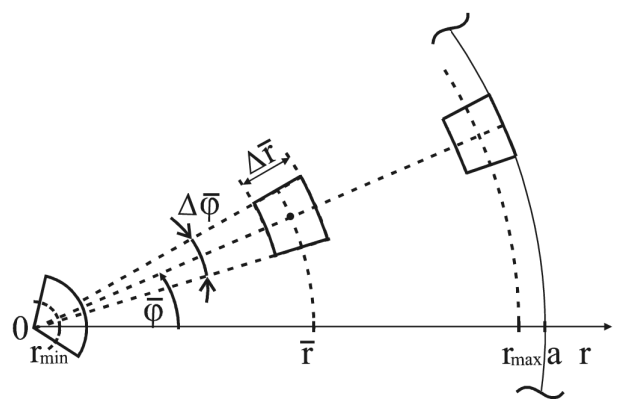

Fig. 2. The location and dimensions of the plate's excitation segment.

The parameters describing the excitations from Eqs. (42) have been chosen to satisfy the following conditions:

$$
\bar{r} \Delta \bar{\varphi}=\Delta \bar{r}, \quad F_{0}=\int_{S} f_{2}\left(r_{0}, \varphi_{0}\right) \mathrm{d} S,
$$

which mean that the plate segment is similar to a square when it is located far from the plate's centre (cf. Fig. 2), and the total force applied to the plate surface is the same for all the excitations. The conditions (43) have been introduced to compare the radiation acoustic power in the case of excitations (42) and can be satisfied for excitations (42c) when $\bar{r} \in\left[\bar{r}_{\min }, \bar{r}_{\max }\right]$ where

$$
\bar{r}_{\min }=\sqrt{S_{0}} / 2, \quad \bar{r}_{\max }=a-\sqrt{S_{0}} / 2 .
$$

The above coordinates determine the most extreme locations of the plate segment. The excitations given by Eqs. (42b) and (42c) have been chosen for practical reasons. They can be useful for modelling of some excitations realised by means of piezoelectric elements located on the plate's surface. The time-harmonic signal applied to the piezoelectric elements causes the changes in their shape and consequently in the plate's vibrations. The point's excitation defined by Eq. (42a) is described by the Dirac delta which simplifies the theoretical analysis of the problem. Therefore, this excitation can be used for modelling the excitations defined by Eqs. (42b) and (42c) 
under some conditions that will be discussed. This allows to simplify the analysis of the acoustic power. Inserting Eqs. (42) into Eqs. (40) yields

$$
\begin{aligned}
& \left\{\begin{array}{l}
f_{1, m, n}^{(c)} \\
f_{1, m, n}^{(s)}
\end{array}\right\}=F_{0}\left\{\begin{array}{l}
W_{m, n}^{(c)}(\bar{r}, \bar{\varphi}) \\
W_{m, n}^{(s)}(\bar{r}, \bar{\varphi})
\end{array}\right\} \\
& \left\{\begin{array}{l}
f_{2, m, n}^{(c)} \\
f_{2, m, n}^{(s)}
\end{array}\right\}=f_{0}\left\{\begin{array}{l}
\delta_{0, m} \\
0
\end{array}\right\} \bar{w}(\bar{a}), \\
& f_{3, m, n}^{(c)}=\frac{f_{0}}{2 \pi}\left\{\begin{array}{c}
\Delta \bar{\varphi}, \quad m=0, \\
(1 / m)[\sin (m(\bar{\varphi}+\Delta \bar{\varphi} / 2)) \\
-\sin (m(\bar{\varphi}-\Delta \bar{\varphi} / 2))], \quad m \geq 1,
\end{array}\right. \\
& f_{3, m, n}^{(s)}=\frac{f_{0}}{2 \pi}\left\{\begin{array}{c}
0, \quad m=0, \\
-(1 / m)[\cos (m(\bar{\varphi}+\Delta \bar{\varphi})) \\
-\cos (m(\bar{\varphi}-\Delta \bar{\varphi})], \quad m \geq 1,
\end{array}\right.
\end{aligned}
$$

where

$$
\bar{w}(b)=\frac{2}{a^{2}} \int_{0}^{b} W_{m, n}(r) r \mathrm{~d} r, \quad 0<b \leq a .
$$

The total acoustic power can be obtained taking into account the finite number of terms in the infinite series from Eq. (36). Consequently, the finite number of the plate's modes can be applied which makes that the obtained results will be correct only for the upper bounded frequencies. The calculations of the acoustic power require taking into account all the modes whose eigenfrequencies are smaller than the excitation frequency. It is also necessary to apply some additional modes whose eigenfrequencies are greater than the excitation frequency. The total number of the modes applied is dependent on the accepted accuracy and considered frequency range. Equation (36) represents the acoustic power. It contains $(2 \bar{M}+1)^{2} \bar{N}^{2}$ modal coefficients of the acoustic impedance in the case of using all the modes $(m, n)$ where $m \leq \bar{M}, n \leq \bar{N}$ and $\bar{M}, \bar{N} \in \mathbb{N}$. The relations (34) allow to reduce the number of modal coefficients to $\bar{N}(\bar{N}+1)(\bar{M}+1)(2 \bar{M}+1) / 2$ and to shorten the time necessary for numerical calculations. The computational complexity in Eq. (36) depends on the squares of numbers $\bar{M}, \bar{N}$ which determine the maximum number of nodal diameters and nodal circles for the applied modes. The acoustic power has been calculated taking into account the nine lowest modes $(m, n)$, where $m=0,1,2$ and $n=1,2,3$, in the numerical analysis presented. All the values of parameters necessary for the analysis are presented in Table.

The acoustic power has been analyzed in the case of the excitations given by Eqs. (42). The axisymmetric uniform excitation (42b) can be approximated by the point excitation from (42a) in the case of $\bar{r}=0$. It is possible to approximate the excitations from Eq. (42c) by the point excitation applied to the plate's point of the polar coordinates $(\bar{r}, \bar{\varphi})$. This leads to some approximation errors. The relative errors of modulus $|\Pi|$ and cosine $\cos \varphi_{\Pi}$ of phase of the acoustic power $\Pi$ has been determined. The errors have been calculated on the basis of the following
TABLE

The values of the parameters describing the analyzed vibroacoustic system. It has been assumed that the plate is steel and the medium is air.

\begin{tabular}{l|c}
\hline \hline \multicolumn{1}{c|}{ Parameter } & Value \\
\hline plate radius & $a=0.3 \mathrm{~m}$ \\
sound velocity in the medium & $c=340 \mathrm{~m} / \mathrm{s}$ \\
Young's modulus & $E=205 \times 10^{9} \mathrm{~Pa}$ \\
plate thickness & $h=1 \times 10^{-3} \mathrm{~m}$ \\
plate centre relative coordinate & $L_{x}=1.5, L_{y}=2$ \\
plate's internal attenuation factor & $\bar{\eta}=10^{-4} \mathrm{~s}$ \\
Poisson's ratio & $\nu=0.3$ \\
plate density & $\rho=7700 \mathrm{~kg} / \mathrm{m}^{3}$ \\
medium density & $\rho_{0}=1.293 \mathrm{~kg} / \mathrm{m}^{3}$
\end{tabular}

relation:

$$
E=\frac{\left|W_{\text {exact }}-W\right|}{\left|W_{\text {exact }}\right|},
$$

where $W$ is the approximate value, $W_{\text {exact }}$ is the exact value.

The influence of parameter $\bar{r}$ on the acoustic power has been also examined. This parameter determines the distance between the plate's centre and the characteristic point of the plate segment (cf. Fig. 2). The analysis has been performed for some sample frequencies and the excitation given by Eq. (42c). The value of the acoustic power modulus varies significantly with frequency. Therefore, the acoustic power modulus has been normalized by $\Pi_{\max }=\max _{\bar{r} / a \in[0,1]}|\Pi|$. It enables presenting several curves in Fig. 3a which shows that the acoustic power modulus decreases significantly when the parameter $\bar{r}$ grows and is negligibly small in comparison with $\Pi_{\max }$ when the excited segment of the plate is located directly at the plate's edge, i.e. when $\bar{r}=\bar{r}_{\max }$. The relative error resulting from applying the point excitation (42a) instead of the true excitation from Eq. (42c) increases significantly when $\bar{r} \rightarrow \bar{r}_{\max }$ (Fig. 3b).

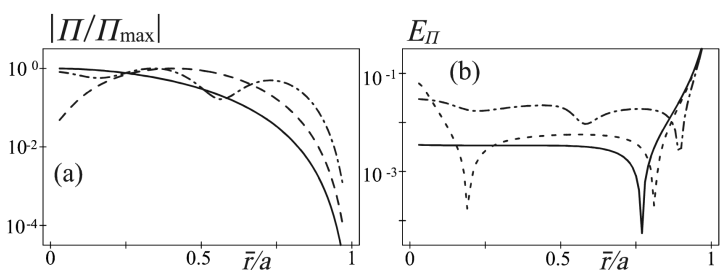

Fig. 3. The normalized acoustic power modulus for excitation (42c) (a) and the relative error resulting from the approximation of excitation (42c) by point excitation (42a) (b). It has been assumed: $\bar{\varphi}=\pi / 4$ and $q=0.001$. Key: solid line $-\omega / \omega_{0,1}=0.5\left(\Pi_{\max }=\right.$ $\left.0.025 \Pi_{0}\right)$, dashed line $-\omega / \omega_{0,1}=2\left(\Pi_{\max }=3.5 \Pi_{0}\right)$, dashed-dotted line $-\omega / \omega_{0,1}=8\left(\Pi_{\max }=0.14 \Pi_{0}\right)$.

The acoustic power has also been calculated for the different values of the quotient $S_{0} / S \in[0.001,0.01]$. The 
acoustic power modulus and phase cosine in the case of the excitations given by Eqs. (42a) and (42c) have been analyzed. The results for both excitations are similar and therefore only the acoustic power in the case of point excitation have been illustrated. The normalized modulus and phase cosine of acoustic power have been presented in Fig. $4 \mathrm{a}$ and $\mathrm{b}$ in the case of excitation given by Eq. (42a). The acoustic power modulus has been normalized by

$$
\Pi_{0}=\frac{\rho_{0} c F_{0}^{2}}{\rho^{2} h^{2} a^{2} \omega_{0,1}^{2}},
$$

which ensures that the obtained results are valid for any value of the exciting force amplitude $F_{0}$. The relative approximation errors of the modulus and phase cosine of acoustic power have been presented in Fig. 4c and d. Figure 4 shows all the quantities as the functions of the normalized frequency $\omega / \omega_{0,1}$ where $\omega_{0,1}=177.24 \mathrm{~Hz}$ for the values of the parameters shown in Table. Figure $4 \mathrm{c}$ and $d$ shows that the relative errors are constant for the frequencies $\omega<\omega_{0,1}$ and for the fixed quotient $S_{0} / S$. The only exception appears within the narrow frequency range around the first eigenfrequency where the relative error of the phase cosine is very small. The values of analyzed errors increase with frequency when $\omega>\omega_{0,1}$. This fact results from the increase of the relative size of the plate's segment in comparison with the acoustic wave length. In the case of $\omega<\omega_{0,1}$, the approximation errors are less than $0.7 \%$ when $S_{0} / S=0.002$ and does not exceed $0.35 \%$ when $S_{0} / S=0.001$.

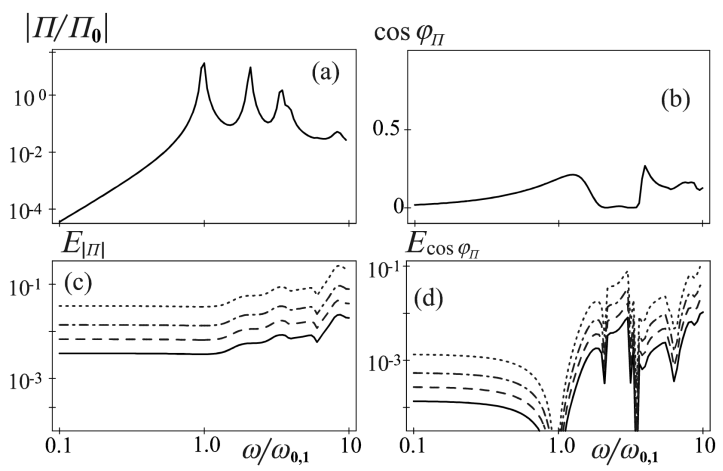

Fig. 4. The acoustic power for point excitation (42a) and the relative errors resulting from the approximation of excitation (42c) by point excitation (42a): (a) normalized modulus, (b) phase cosine, (c) modulus relative errors, (d) phase cosine relative errors. Key: solid line $-q=0.001$, dashed line $-q=0.002$, dashed-dotted line $-q=0.004$, dotted line $-q=0.01$. It has been assumed: $\bar{r}=0.5 a, \bar{\varphi}=\pi / 4$.

The normalized modulus and the phase cosine of acoustic power have been presented in Fig. $5 \mathrm{a}$ and $\mathrm{b}$ together with their relative approximation errors. The analysis has been performed for the point excitation given by Eq. (42a) and for some sample values of parameter $\bar{r}$ including the case when the excited plate's segment is located in the smallest possible distance from the plate's centre i.e. $\bar{r}=\bar{r}_{\text {min }}$. Since the acoustic power achieves very small value when the excited plate segment is located directly at the plate's edge, this case has not been analyzed. The relative approximation error does not exceed $0.25 \%$ for $\omega<\omega_{0,1}$ and $S_{0} / S=0.001$. The excitation applied to the plate's segment defined by Eq. (42c) can be approximated by the point excitation with an approximation error less than $0.36 \%$ in the case when $S_{0} / S=0.001, \omega<\omega_{0,1}$ and $\bar{r} \in(0,0.75 a]$ (Fig. 5c,d).

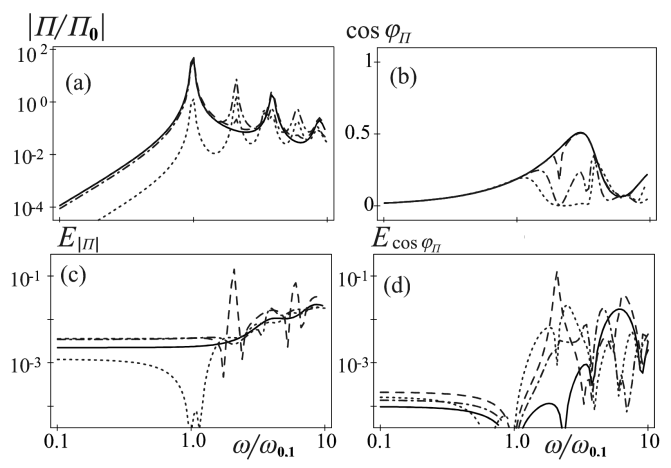

Fig. 5. The acoustic power for excitation (42a) and the relative errors resulting from the approximation of excitations (42b) and (42c) by point excitation (42a) (a) normalized modulus, (b) phase cosine, (c) modulus relative errors, and (d) phase cosine relative errors. Key: solid line $-\bar{r}=0$, dashed line $-\bar{r}=0.03 a$, dashed-dotted line $-\bar{r}=0.25 a$, dotted line $-\bar{r}=0.75 a$. It has been assumed: $\bar{\varphi}=\pi / 4, q=0.001$.

This is obvious that the smaller area $S_{0}$ to which excitation is applied the smaller the approximation error resulting from using the point excitation instead of excitations (42b) or (42c). However such approximation is useful for practical reason and therefore this error has been shown in Fig. 6 as a function of the quotient $S_{0} / S$. This figure confirms the above conclusion. The analysis has been performed for the different locations of the excited plate's segment and for some sample frequencies. In the case of $\omega>\omega_{0,1}$, the curves presented in Fig. $6 \mathrm{~b}$ concern the frequencies for which the maximum values of error are observed. Figure 6 shows that the presented error varies linearly with $S_{0} / S$ when it is depicted on the full logarithmic scale.

The acoustic attenuation can be neglected under some conditions, by assuming $\varepsilon_{0}=0$ in equations system (41), to obtain simple formulae describing the values of constants $c_{m, n}^{(c)}, c_{m, n}^{(s)}$ and to simplify the numerical calculation of the acoustic power. The negligence of the acoustic attenuation causes, however, an approximation error of value depending on the normalized excitation frequency $\omega / \omega_{0,1}$. The relative approximation error of the modulus and the phase cosine of the acoustic power have been presented in Fig. 7. It can be noticed that the modulus error can assume significant values whereas the error of the phase is negligible. Both errors grow rapidly with frequency when $\omega<\omega_{0,1}$. The negligence of the acoustic 


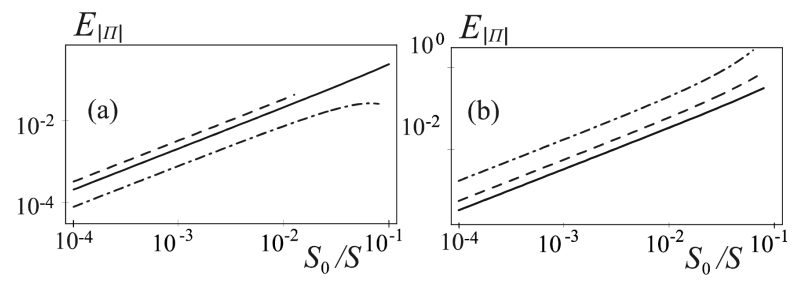

Fig. 6. The relative errors of the acoustic power modulus $|\Pi|$, for $\bar{\varphi}=\pi / 4$, resulting from the approximation of excitation (42b) and (42c) by the point excitation (42a): (a) $\omega / \omega_{0,1}=0.5$, (b) $\bar{r}=0.25 a$. Key: (a) solid line $-\bar{r}=0$, dashed line $-\bar{r}=0.1 a$, dashed-dotted line $-\bar{r}=0.75 a$, (b) solid line $-\omega / \omega_{0,1}=0.1$, dashed line $-\omega / \omega_{0,1}=3$, dashed-dotted line $-\omega / \omega_{0,1}=4$.

attenuation causes the relative error of the acoustic power modulus less than $1 \%$ only within the low frequency range for $\omega<0.3 \omega_{0,1}$ which means that for frequencies higher than $0.3 \omega_{0,1}$ the acoustic attenuation must be included to assure the desirable computation accuracy. If the $10 \%$ error is acceptable then the acoustic attenuation can be neglected within the entire frequency band. Additionally, the error achieves some significant values at the resonance frequencies.

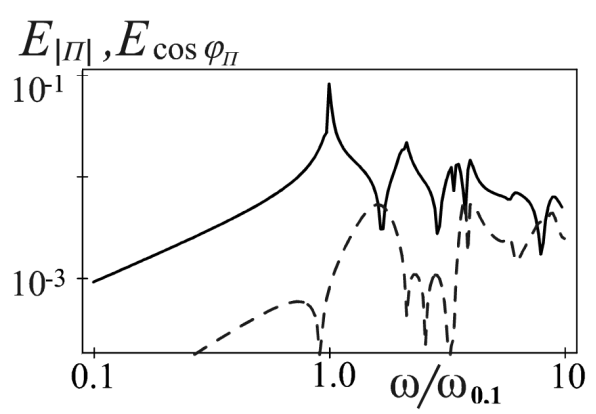

Fig. 7. The relative approximation errors of the acoustic power resulting from the negligence of the acoustic attenuation for the excitation given by Eq. (42a), $\bar{r}=0.5 a$ and $\bar{\varphi}=\pi / 4$. Key: solid line - the modulus errors, dashed line - the phase cosine errors.

It is essential to determine the influence of the transverse baffles on the acoustic power of the considered sound source. The following quantity has been introduced for this purpose:

$$
\delta \Pi=\frac{|\Pi|-|\bar{\Pi}|}{|\bar{\Pi}|},
$$

where $\bar{\Pi}$ is given in Eq. (A.1) and denotes the acoustic power of a clamped circular plate located in a flat baffle. This quantity is the normalized measure of the influence of transverse baffles on the acoustic power modulus. The case when $\delta \Pi>0$ means an increase in the modulus $|\Pi|$ resulting from the existence of the additional baffles and the case when $\delta \Pi<0$ indicates the modulus reduction. The quantities $\delta \Pi$ and $\bar{\Pi}$ have been pre- sented in Fig. 8 as functions of the normalized frequency $\omega / \omega_{0,1}$. The quantity $\delta \Pi$ has been shown for the different distances between the plate's centre and the transverse baffles. The point excitation given in Eq. (42a) has been assumed. Figure 8 shows that the transverse baffles cause an increase in the acoustic power modulus for frequencies $\omega<\omega_{0,1}$. It can also be noticed, for these frequencies, that the baffles influence grows when the distance between the plate's centre and the baffles decreases i.e. when $L \rightarrow 1$. The greater influence is observed for $\omega<\omega_{0,1}$. In the case of $\omega>\omega_{0,1}$, the influence decreases with an increase in frequency. This is due to the growth of quotients $l_{x} / \lambda$ and $l_{y} / \lambda$ which means that the distance between the source and baffles grows in comparison with the wavelength. The influence depends strongly on frequency when $\omega / \omega_{0,1} \in(0.9,1.0)$. The phase depends very weakly on the presence of the baffles within the entire frequency range and for any values of quotients $l_{x} / \lambda$ and $l_{y} / \lambda$.

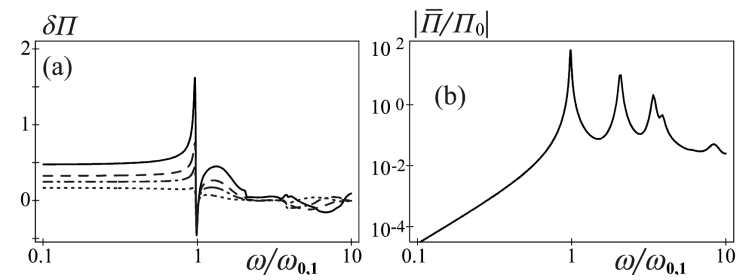

Fig. 8. The influence of the transverse baffles on the acoustic power for the excitation given by Eq. (42a), $\bar{r}=0.5 a$ and $\bar{\varphi}=\pi / 4$ : (a) the quantity $\delta \Pi$ defined by Eq. (51), (b) the normalized modulus of the acoustic power radiated by the plate located in a flat baffle. Key: solid line $-L_{x}=L_{y}=1$; dashed line $-L_{x}=L_{y}=$ 1.5, dashed-dotted line $-L_{x}=L_{y}=2$, dotted line $L_{x}=L_{y}=3$.

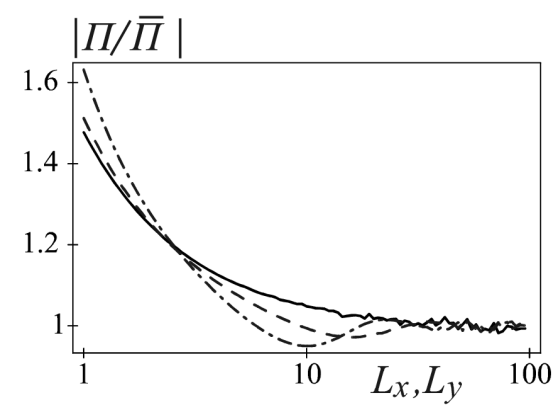

Fig. 9. The normalized modulus of the acoustic power $|\Pi / \bar{\Pi}|$ for the excitation given by Eq. (42a), $\bar{r}=$ $0.5 a, \bar{\varphi}=\pi / 4$ and $L_{x}=L_{y}$. Key: solid line $\omega / \omega_{0,1}=0.1\left(\bar{\Pi}=27.8 \times 10^{-6} \Pi_{0}\right) ;$ dashed line $\omega / \omega_{0,1}=0.5\left(\Pi=0.6 \times 10^{-2} \Pi_{0}\right)$, dash-dotted line $\omega / \omega_{0,1}=0.8\left(\bar{\Pi}=0.11 \times \Pi_{0}\right)$.

The normalized quantity $|\Pi / \bar{\Pi}|$, as a measure of the baffles influence on the acoustic power, has been included. This quantity has been shown in Fig. 9 as a function of 
the distance between plate's centre and the transverse baffles in the case when $L_{x}=L_{y}$. The analysis has been performed for some sample frequencies less than $\omega_{0,1}$. It has been shown that $|\Pi / \bar{\Pi}|$ assumes some significant values for $L_{x}, L_{y}<31$. It grows with the frequency when $L_{x}, L_{y}<2.5$. This fact results from the interference of the waves derived directly from the plate and the waves reflected from the baffles. This interference is significant when the plate is located in the vicinity of the baffles. Moreover, the baffles influence decreases when the distances $L_{x}$ and $L_{y}$ between the plate's centre and the baffles grow which is in agreement with expectations. The baffles influence decreases the faster the higher frequency.

\section{Concluding remarks}

The theoretical and numerical analyses have been carried out leading to the conclusion that the additional transverse baffles influence significantly the radiated acoustic power within the low frequency range. Consequently, this influence cannot be neglected for the low frequencies which is especially important in actual active noise and vibration control problems. The influence of the transverse baffles decreases when the vibration frequency grows. Moreover, it has also been noticed that the greatest influence of the baffles on the acoustic power is when the vibrating source is located close to the baffles. When the distance between the source and the baffles increases the baffles influence decreases. The obtained results indicate some situations for which the baffles impact should be taken into account and some situations for which that impact can be neglected. The influence of a uniform excitation located on the plate fragment of the small area can be closely approximated by the point force excitation in the case of the low frequency range. That fact enables to simplify theoretical calculation and reduces the numerical calculation time. Therefore, modelling uniform excitations by means of the point force excitation can be used to improve algorithms employed in the case of noise control. The acoustic power achieves its maximum when the excited segment of the plate is located near the source's centre.

\section{Appendix. The acoustic power of clamped circular plate located in a flat baffle}

On the basis of Eqs. (32), (35), (36) and (41), the total acoustic power of clamped circular plate located in a flat baffle can be obtained. For this purpose, it has been assumed that the transverse baffles are located infinitely far from the plate centre which can be written as $L_{x}, L_{y} \rightarrow \infty$. Then, applying the relation $\lim _{u \rightarrow \infty} J_{\hat{m}}(u)=0$ for $\hat{m}=0,1,2, \ldots$ (cf. [21]) results in

$$
\bar{\Pi}=\frac{1}{2} S \rho_{0} c \omega^{2} \sum_{m=0}^{\infty} \sum_{n=1}^{\infty} \sum_{l=1}^{\infty}\left[\bar{c}_{m, n}^{(c)} \bar{c}_{m, l}^{*(c)}\right.
$$

$$
\left.+\operatorname{sgn}(m) \bar{c}_{m, n}^{(s)} \bar{c}_{m, l}^{*(s)}\right] \zeta_{m ; n, l}
$$

where $\bar{\Pi}$ is the acoustic power of the clamped circular plate located in a flat baffle and

$$
\zeta_{m ; n, l}=8 \beta^{2} \int_{0}^{\infty} \frac{\psi_{m, n}(\tau) \psi_{m, l}(\tau) \tau \mathrm{d} \tau}{\sqrt{1-\tau^{2}}} .
$$

Constants $\bar{c}_{m, n}^{(c)}, \bar{c}_{m, n}^{(s)}$ appearing in Eq. (A.1) can be calculated on the basis of the equations system

$$
\begin{aligned}
& \bar{c}_{m, n}^{(c)}\left(\frac{k_{m, n}^{4}}{k_{\mathrm{T}}^{4}}-1\right)-\mathrm{i} \varepsilon_{0} \frac{\omega_{0,1}}{\omega} \sum_{l=1}^{\infty}\left(\bar{c}_{m, l}^{(c)} \zeta_{m ; n, l}\right) \\
& =\frac{f_{m, n}^{(c)}}{\rho h \omega^{2}}, \\
& \bar{c}_{m, n}^{(s)}\left(\frac{k_{m, n}^{4}}{k_{\mathrm{T}}^{4}}-1\right)-\mathrm{i} \varepsilon_{0} \frac{\omega_{0,1}}{\omega} \sum_{l=1}^{\infty}\left(\bar{c}_{m, l}^{(s)} \operatorname{sgn}(m) \zeta_{m ; n, l}\right) \\
& =\frac{f_{m, n}^{(s)}}{\rho h \omega^{2}} .
\end{aligned}
$$

The quantity $\zeta_{m ; n, l}$ defined by Eq. (A.2) is the modal coefficient of acoustic impedance of a clamped circular plate located in a flat baffle. This coefficient describes the interactions of both modes, cosine-cosine and sinesine. Moreover, it is worth noticing that in the case of the quantity the both interacting modes are described by the same number of nodal diameters. The acoustic power of the clamped circular plate located in a flat baffle has been expressed by the triple series containing the modal and it is less computationally complex than the quantity given in Eq. (36) which represents the acoustic power of the clamped circular plate located at the boundary of the three-wall corner region.

\section{References}

[1] J.P. Arenas, M.J. Crocker, Int. J. Acoust. Vibrat. 7, 217 (2002).

[2] J.P. Arenas, Int. J. Occupat. Safety Ergonom. 15, 401 (2009).

[3] D. Zou, M.J. Crocker, Arch. Acoust. 34, 25 (2009).

[4] W.P. Rdzanek, W.J. Rdzanek, K. Szemela, J. Comput. Acoust. 18, 1 (2010).

[5] S. Alper, E.B. Magrab, J. Acoust. Soc. Am. 48, 681 (1970).

[6] H. Levine, F.G. Leppington, J. Sound Vibrat. 121, 269 (1988).

[7] W.P. Rdzanek, Arch. Acoust. 16, 581 (1991).

[8] W.P. Rdzanek, W.J. Rdzanek, Z. Engel, K. Szemela, Int. J. Occupat. Safety Ergonom. 13, 147 (2007).

[9] P. Kos, L. Leniowska, Arch. Acoust. 32, 895 (2007).

[10] J. Wiciak, Arch. Acoust. 32 (Suppl.), 265 (2007).

[11] A. Branski, S. Szela, Arch. Acoust. 33, 521 (2008).

[12] K.T. Chen, S.H. Chang, C.H. Chou, Y.H. Liu, Appl. Acoust. 69, 367 (2008). 
[13] M. Kozupa, J. Wiciak, Acta Phys. Pol. A 118, 95 (2010).

[14] D. Zou, M.J. Crocker, Arch. Acoust. 34, 13 (2009).

[15] W.J. Rdzanek, W.P. Rdzanek, Arch. Acoust. 31, (Suppl.), 99 (2006).

[16] W.P. Rdzanek, K. Szemela, D. Pieczonka, Arch. Acoust. 32, 883 (2007).

[17] W.P. Rdzanek, K. Szemela, Arch. Acoust. 32, 339 (2007).

[18] W.P. Rdzanek, W.J. Rdzanek, K. Szemela, Hydroacustics Ann. J. 11, 339 (2008).

[19] W.P. Rdzanek, W.J. Rdzanek, K. Szemela, Arch. Acoust. 34, 75 (2009).
[20] L. Meirovitch, Analytical Methods in Vibrations, Macmillan, New York 1967.

[21] N.W. McLachlan, Bessel Functions for Engineers, Clarendon Press, Oxford 1955.

[22] A.W. Leissa, Vibration of Plates, Vol. SP-160, NASA, U.S. Government Printing Office, Washington 1969.

[23] E. Skudrzyk, Simple and Complex Vibration Systems, Pennsylvania State University Press, London 1968.

[24] P.M. Morse, K.U. Ingard, Theoretical Acoustics, Princeton University Press, Princeton 1968.

[25] P.M. Morse, Vibration and Sound, AIP, New York 1995. 